\title{
Determination of Phosphate using a Highly Sensitive Paired Emitter-Detector Diode Photometric Flow
} Detector

\author{
Martina O' Toole, King Tong Lau, Roderick Shepherd, Conor Slater and Dermot \\ Diamond ${ }^{*}$ \\ Adaptive Sensors Group, National Centre for Sensor Research, School of Chemical \\ Sciences, Dublin City University, Dublin 9.
}

\begin{abstract}
The use of a novel inexpensive photometric device, Paired Emitter Detector Diode (PEDD) has been applied to the colorimetric determination of phosphate using the malachite green spectrophotometric method. The novel miniaturized flow detector applied within this manifold is a highly sensitive, low cost, miniaturized light emitting diode (LED) based detector. The optical flow cell was constructed from two LEDs, whereby one is the light source and the second is the light detector, with the LED light source forward biased and the LED detector reversed biased. The photocurrent generated by the LED light source discharges the junction capacitance of the detector diode from 5 $\mathrm{V}$ (logic 1$)$ to $1.7 \mathrm{~V}(\operatorname{logic} 0)$ and the time taken for this process to occur is measured using a simple timer circuit.
\end{abstract}

\footnotetext{
${ }^{*}$ Correspondence author: Tel.: +353-1-7005404; fax: +353-1-7008002 email: dermot.diamond@dcu.ie
} 
The malachite green (MG) method employed for phosphate determination is based on the formation of a green molybdophosphoric acid complex, the intensity of which is directly related to phosphate concentration. Optimum analytical parameters such as reaction kinetics, reagent to sample concentration ratio and emitter wavelength intensity were investigated for the spectrophotometric method. Linear calibration plots that obeyed the Beer-Lambert Law were obtained for phosphate in the range of $0.02-2 \mu \mathrm{M}$. The dynamic range, sensitivity and limits of detection are reported.

Keywords: Light Emitting Diode Detector, Malachite Green, Phosphate, Colorimetric Flow Analysis.

\section{Introduction}

Phosphorus, specifically orthophosphate, is an essential nutrient used by plants and animals for growth and energy transport [1]. However elevated concentrations in aquatic ecosystems cause the phenomenon of eutrophication, which can result in algal bloom formation [2]. The rapid growth of aquatic vegetation in turn causes the death and decay of vegetation and aquatic life due to the decrease in dissolved oxygen levels. The resulting eutrophication of natural waters is a subject of utmost concern, and has been recognized by the European Union through legislation that stipulates $0.1 \mathrm{mg} \mathrm{L}^{-1} \mathrm{PO}_{4}$ as an indicator level for possible problematic algal growth in rivers [3].

Spectrophotometric procedures for monitoring orthophosphate include the molybdenum blue method [1,3-7], the yellow vanadomolybdate complex method [8-11] and the 
malachite green method [12-17]. The malachite green method has been shown to enhance sensitivity by approximately 4 times when compared to the aforementioned methods $[12,18]$. A significant advantage of the malachite green method is its lack of sensitivity to experimental conditions such as changes in heating, reagent addition sequence or reaction time [12,19]. Additional advantages include higher sensitivity compared to the molybdenum blue method and the longer optimum detection wavelength than the yellow method [20]. The malachite green method is based on the reaction at low $\mathrm{pH}$ between ammonium molybdate, polyvinyl alcohol (PVA) and malachite green (MG) as in the following equations [20]:

$$
\begin{aligned}
& \mathrm{H}_{3} \mathrm{PMo}_{12} \mathrm{O}_{40}+\mathrm{HMG}^{2+} \quad \rightarrow \quad\left(\mathrm{MG}^{+}\right)\left(\mathrm{H}_{2} \mathrm{PMo}_{12} \mathrm{O}_{40}^{-}\right)+2 \mathrm{H}^{+} \\
& \text {(yellow) (yellow, } \left.\left.\lambda_{\max } 446 \mathrm{~nm}\right) \quad \text { (green, } \lambda_{\max } 640 \mathrm{~nm}\right) \\
& \left(M G^{+}\right)\left(H_{2} P M o_{12} O_{40}^{-}\right)+H M G^{2+} \rightarrow\left(M G^{+}\right)_{2}\left(H P M o_{12} O_{40}^{2-}\right)+2 H^{+} \\
& \left(M G^{+}\right)_{2}\left(H P M o_{12} O_{40}^{2-}\right)+H M G^{2+} \rightarrow\left(M G^{+}\right)_{3}\left(P M o_{12} O_{40}^{3-}\right)+2 H^{+}
\end{aligned}
$$

In the presence of large excess of MG, the reactions (1-3) can occur, and the 3:1 ion associate formed in Eq. 3 can easily precipitate in the acidic aqueous solution. To prevent the formation of the ion association reactions shown in Eqs. 2 and 3, and to stabilize the ion associate in the aqueous solution PVA is added to the solution [14,20].

The optical detection employed in this research to determine phosphates via the malachite green method is a novel, highly sensitive, low cost paired emitter-detector diode (PEDD) 
photometric detector. The PEDD flow cell consists of two LEDs, whereby one is the light source and the second is the light detector. The LED light source is forward biased while the LED detector is reversed biased. The photon flux from the emitter LED strikes the detector LED, generating a small photocurrent (of the order of nanoampere) that discharges the capacitor voltage over time. The photocurrent produced is not measured directly as this would require an expensive nanoamperometer. Instead, the parameter monitored is the decay time $(\mu \mathrm{s})$ taken for the discharge process to go from an initial value of $5 \mathrm{~V}(\operatorname{logic} 1)$ to a preset value of $1.7 \mathrm{~V}(\operatorname{logic} 0)$ using a simple timer circuit, and a comparator which determines whether the remaining charge is above or below the set point $(+1.7 \mathrm{~V})$. The use of LEDs offer advantages such as low cost, compact form, availability across a broad spectral range from UV to near-IR, robust and long lifetimes [21-23]. In most microanalytical systems the light source and the photodetector are normally separate units integrated with the microfluidic manifold [24], however in this case the PEDD is a single unit containing light source, fluid channel and detector for flow analysis [25-27].

In this paper we present the detection of phosphates using this novel detector and a comparative study carried out using both a photodetector comprising of an LED as a light source and a photodiode as the detector and a commercially available platewell reader. Optimum conditions such as time allowed for colour formation, reagent to sample ratio and emitter wavelength and intensity were investigated for the spectrophotometric method. The dynamic range, sensitivity, limits of detection and linear range were determined. Under optimised conditions the low cost PEDD detector ( $\sim \$ 1)$ displayed higher sensitivity and improved precision compared to the commonly employed LED- 
photodiode detector and a commercially available platewell reader. As such it could provide a route to autonomous, very low cost, low power consuming, highly sensitive, field deployable analytical measurements, which would form the basis of widely deployed chemosensor networks [28].

\section{Experimental}

\subsection{Chemicals and Reagents}

All solutions were prepared from analytical grade chemicals. Deionised water obtained from a Millipore Milli-Q water purification system was used for all analysis. A stock malachite green reagent was prepared by slowly adding $100 \mathrm{~mL}$ concentrated sulphuric acid $\left(\mathrm{H}_{2} \mathrm{SO}_{4}, 98 \%\right.$, Fisher Scientific UK Ltd $)$ to approximately $400 \mathrm{~mL}$ of deionised water. The solution was allowed to cool to room temperature before adding $27 \mathrm{~g}$ of ammonium molybdate $\left(\left(\mathrm{NH}_{4}\right)_{6} \mathrm{Mo}_{7} \mathrm{O}_{24} .7 \mathrm{H}_{2} \mathrm{O}\right.$, Fluka, Dublin, Ireland). Malachite green oxalate $\left(\mathrm{C}_{25} \mathrm{H}_{22} \mathrm{~N}_{2} \mathrm{O}_{4}\right)$ (Baltimore Biological Laboratory, Baltimore, Md.) (0.135 g) was then added to the solution and stirred until dissolved. The solution was then made up to 1 L, vacuum filtered $(0.45 \mu \mathrm{m}$ Nylaflo®, VWR International, Meath, Ireland) and stored at $4{ }^{\circ} \mathrm{C}$. A stock solution of $0.1 \%$ (w/v) polyvinyl alcohol (PVA) (Sigma Aldrich, Dublin, Ireland) was prepared by dissolving $5 \mathrm{~g}$ in $500 \mathrm{~mL}$. To assist the dissolution process, the solution was heated to near boiling point while being stirred continuously. Both stock

solutions were stored in the dark at $4{ }^{\circ} \mathrm{C}$. The colour reagent was prepared daily by mixing equal amounts of each stock reagent. Standard solutions of phosphorus (P) were prepared daily from a stock solution of $1 \mathrm{mM}$ potassium phosphate dibasic $\left(\mathrm{K}_{2} \mathrm{HPO}_{4}\right.$, 
Sigma Aldrich, Dublin, Ireland). The stock solution was prepared by dissolving $0.0175 \mathrm{~g}$ $\mathrm{K}_{2} \mathrm{HPO}_{4}$ in $100 \mathrm{~mL}$ deionised water. A new stock solution was prepared weekly.

\subsection{Measurement Procedure}

The colorimetric reagent $(1 \mathrm{~mL})$ was added to the samples $(6 \mathrm{~mL})$ and the solution was left to stand for 30 minutes for colorimetric development. The absorbance of the solution was measured comparatively using the $\mu \mathrm{Quant}{ }^{\mathrm{TM}}$ platewell reader (Bio-Tek Instruments, Inc., USA), the LED-photodiode $\left(\lambda_{\max } 636 \mathrm{~nm}\right)$ photodetector and the PEDD flow cell $\left(\lambda_{\max } 636 \mathrm{~nm}\right)$. The colorimetric reagent was prepared daily by mixing equal amounts of the malachite green and PVA reagents.

\subsection{Fabrication of PEDD optical flow cell and the LED-photodiode detector}

The integrated PEDD cell was fabricated as previously described [25] using two $5 \mathrm{~mm}$ LEDs (Kingbright, Ireland) as shown in Fig. 1.

The detector used was a red LED $\left(\lambda_{\max }\right.$ at $\left.660 \mathrm{~nm}\right)$ which can detect any wavelength below this point. A red LED ( $\lambda_{\max }$ at $\left.636 \mathrm{~nm}\right)$ was used as the emitter LED.

The LED-photodiode detector was fabricated similarly to that of the PEDD illustrated in Fig. 1. A red LED ( $\lambda_{\max }$ at $636 \mathrm{~nm}$ ) was used as the emitter LED with a pathlength of 1.3 $\mathrm{mm}$. The LED detector was replaced with a Si photodiode, IPL10020BW (Thales Optronics, UK) detector and bonded in the same way as the PEDD. 


\subsection{Light Measurement}

A $9 \mathrm{~V}$ battery was used as the power source and this was regulated to a stable $5 \mathrm{~V}$ using a voltage regulator. The control circuitry used to drive and monitor the PEDD flow detector was operated from the regulated $5 \mathrm{~V}$ supply. The light detector LED in output mode was reverse biased at $5 \mathrm{~V}$ for $100 \mu$ s and then switched to input mode. Photons emitted from the red emitter LED strike the red detector LED to generate a small photocurrent, which in turn discharges the detector LED. The time taken for the discharge process to go from an initial value of $5 \mathrm{~V}$ (logic 1) to a preset value of $1.7 \mathrm{~V}$ (logic 0) was measured with a simple timer circuit.

The circuitry used to drive the LED photodiode detector is outlined in Fig. S1.

Fig. S1 shows a light emitting diode (D1) illuminating a photodiode (D2) connected to a current to voltage converter. The voltage between $\mathrm{V}_{\text {out }}$ and GND is then measured by an analogue digital converter on a microcontroller. The current through the LED is adjusted by a potentiometer (R1) thus controlling the illumination. The output current of a photodiode in reverse bias is linearly proportional to the light intensity. To condition the signal for the 10 bit ADC on a PIC16F876 microcontroller a current to voltage converter is employed. A circuit consisting of a FET input operational amplifier (IC1) and a resistor (R2) in feed back was constructed.

In the circuit the output voltage is determined by Eq. 4:

$\mathrm{V}_{\text {out }}=-\mathrm{I}_{\mathrm{p}} \times \mathrm{R} 2$,

Whereby output voltage is $\mathrm{V}_{\text {out }}, \mathrm{I}_{\mathrm{p}}$ is the current through the photodiode and R2 is a 40 $\mathrm{M} \Omega$ resistor. A capacitor $(\mathrm{C} 1)$ is not a requirement but was added to reduce noise. 
Although a circuit with enhanced performance can be constructed, the cost incurred for slight increases in sensitivity by using more precise components and/or higher resolution ADCs would not be justified on a low cost sensor. The reported absorbance (A) measurement was calculated according to Eq. 5:

$A=-\log \left(\frac{\text { SampleIntensity }}{\text { BlankIntensity }}\right)$

Where Sample Intensity is the intensity of the light passing through the cell with sample solution and Blank Intensity is the intensity of the light passing through the cell with reference solution.

\section{Results and Discussion}

\subsection{Absorption Spectrum}

The measurement is based on the following theoretical model (Eq. 6), which has been derived by Lau et al. [29].

$\log (t)=\varepsilon C l+\log \left(t_{0}\right)$

Where 1 is the optical pathlength through the solution $(\mathrm{cm}), \varepsilon$ the molar extinction coefficient, $\mathrm{C}$ the concentration of the absorbing species $\left(\mathrm{molL}^{-1}\right), \mathrm{t}_{0}$ a constant that represents discharge time in the absence of the coloured species in solution ( $\mu$ s) and $t$ is the discharge time in the presence of the coloured species in solution $(\mu \mathrm{s})$. 
The optimum wavelength to monitor the malachite green-molybdphosphate complex has been variously reported in the literature, typically citing the $\lambda_{\max }$ in the range ca. 600 to $650 \mathrm{~nm}[12,13,30]$. We therefore determined the $\lambda_{\max }$ under our experimental conditions by obtaining the absorbance spectrum of $0.9 \mu \mathrm{M} \mathrm{PO}_{4}$ using the $\mu$ Quant ${ }^{\mathrm{TM}}$ platewell reader (Bio-Tek Instruments, Inc., USA). The $\lambda_{\max }$ of the sample was found to be $640 \mathrm{~nm}$. We therefore selected an emitter LED with a $\lambda_{\max }$ of $636 \mathrm{~nm}$. The light intensity transmitted from the emitter LED $\left(\lambda_{\max } 636 \mathrm{~nm}\right)$ was measured with a detector LED that had a slightly smaller bandgap $\left(\lambda_{\max } 660 \mathrm{~nm}\right)$. The absorbance of the malachite greenmolybdphosphate species $\left(\lambda_{\max } 640 \mathrm{~nm}\right)$ as shown in Fig. 2 efficiently overlaps with the emission spectrum of the emitter red LED and will therefore allow high sensitivity. The emission spectrum of the emitter LED was obtained by using Ocean Optics spectrometer (OOIBase 32 ${ }^{\mathrm{TM}}$, Ocean Optics, Inc., Dunedin, USA).

\subsection{Optimisation of standard procedure}

The optimum reagent to sample ratio was determined by preparing a range of samples at a concentration of $2 \mu \mathrm{M} \mathrm{PO}_{4}$ with varying volumes of reagent $(0.1-2 \mathrm{~mL})$ added. The normalized maximum absorbance $\left(\mathrm{R} / \mathrm{R}_{\max }\right)$ of each sample was recorded after 40 minutes and plotted against the volume of reagent added to $6 \mathrm{~mL}$ of sample, see Fig. S2.

As shown in Fig. $\mathrm{S} 2$ the reagent volume that provides the highest $R / R_{\max }$ i.e. the most intense colour was the $6: 1 \mathrm{v} / \mathrm{v}$ sample to reagent ratio (i.e. $6 \mathrm{~mL}$ of sample to $1 \mathrm{~mL}$ reagent). This was the sample to reagent ratio adopted throughout all remaining experiments. 
Previous studies have shown the need to optimise the emitter LED light intensity [25,26], as the change in discharge time (i.e. the resolution) can be improved by up to a factor of 8. As LEDs vary with regard to their light intensity it was necessary to determine the optimum resistance required for a $636 \mathrm{~nm}$ LED. The optimization of the light intensity was carried out as previously described using a $(0-10 \mathrm{k} \Omega)$ variable resistor [26]. A resistance of $1.58 \mathrm{k} \Omega$ was found to provide the optimum light source intensity resulting in high sensitivity, while maintaining a smooth baseline without drift. A 12-pt moving average has been applied to the data shown in Fig. S3. The effects of decreasing the light intensity on the response are clearly demonstrated in Fig. S3. For example, at a resistance of $0.004 \mathrm{k} \Omega$, the difference in discharge time $(\Delta \mathrm{t})$ i.e. peak height was $4.78 \pm 0.19 \mu \mathrm{s}$ with an R.S.D. $(n=3)$ of $3.90 \%$. At an additional applied resistance of $1.58 \mathrm{k} \Omega$ the peak height obtained was $32.59 \pm 0.44 \mu$ s with $1.35 \%$ R.S.D. $(n=3)$. The standard deviation $(n=3)$ of the baseline was $6.95 \mu \mathrm{s}$. At a resistance of $6.02 \mathrm{k} \Omega$ the peak height achieved was $144.87 \pm 0.78 \mu$ s with $0.54 \%$ R.S.D. $(n=3)$. Increasing the resistance to $6.02 \mathrm{k} \Omega$ further improved the change in discharge time but the standard deviation $(n=3)$ of the baseline deteriorated to $29.37 \mu \mathrm{s}$.

Further increases in resistance increased the peak height even more, but caused more baseline drift and higher R.S.D. values as shown in Fig. S3. Applying a moving average to the data set can compensate for the increase in baseline noise and drift. An additional disadvantage however of over-increasing the emitter resistance is that the resulting decrease in emitted light intensity can lead to a reduction in the dynamic range. 


\subsection{Kinetics Study}

Linge et al. [12] reported a stand time for sample and reagent colour development of approximately 30 minutes. The development of the malachite green-molybdphosphate colour intensity with $0.5 \mu \mathrm{M} \mathrm{PO}_{4}$ was monitored using the $\mu$ Quant ${ }^{\mathrm{TM}}$ platewell reader by taking an absorbance measurement every 2 minutes.

As shown in Fig. 3 the colour formation increased rapidly until approximately 27 minutes after which the rate of increase decreased to a much slower stage. This was in agreement with the time allowed for colour developed outlined by Linge et. al [12].

\subsection{Phosphate measurement using PEDD flow cell, LED photodiode flow cell and the $\mu Q u a n t^{\mathrm{TM}}$ platewell reader}

Working calibration solutions between 0.002 and $20 \mu \mathrm{M}$ were prepared from the stock standard. The malachite green method has a limited range of up to $20 \mu \mathrm{M}$ before precipitation of MG occurs. Various concentrations of phosphate were prepared in deionised water and passed through the PEDD flow cell for ca. 4 minutes per sample, at a flow rate of $0.6 \mathrm{mLmin}^{-1}$. The $\log$ of the discharge times $(\log \mathrm{t}, \mu \mathrm{s})$ was plotted against malachite green-molybdphosphate complex concentration (C) in accordance with the model (Eq. 6) and the result is presented in Fig. 4. The inset plot Fig. 4 shows a large dynamic range from ca. 0 to $20 \mu \mathrm{M}$ malachite green-molybdphosphate complex from which a linear range of approximately $0.02-2 \mu \mathrm{M}$ malachite green-molybdphosphate $\left(\mathrm{R}^{2}\right.$ value 0.9964$)$ was observed as shown in main feature plot. The relative standard 
deviation of the measurements ( $n=3$, shown as error bars) is very low (ca. $0.05 \%)$ and a LOD of ca. $2 \mathrm{nM}$ of the malachite green-molybdphosphate complex as shown in Fig 5.

An LOD of $2 \mathrm{nM}$ was achieved using the PEDD $\left(\lambda_{\max } 636 \mathrm{~nm}\right)$. The response (change in discharge time, $\mu$ s) obtained was $8.49 \pm 0.82 \mu$ s with an RSD of $9 \%(n=3)$.

As a comparison study, the absorbance of the same malachite green-molybdphosphate complex concentrations were acquired employing both the commonly used LEDphotodiode detector and a $\mu$ Quant ${ }^{\mathrm{TM}}$ platewell reader.

As shown in Fig. S4 the mean normalized maximum absorbance $\left(R / R_{\max }\right)$ was plotted against malachite green-molybdphosphate complex concentration (C) resulting in a dynamic range of $0.2-20 \mu \mathrm{M}$. A linear range of $\left(\mathrm{R}^{2}\right.$ value 0.9947$)$ of $0.2-2 \mu \mathrm{M}$ was achieved, with an R.S.D. $(n=3)$ of $8.7 \%$. A significantly higher LOD of $0.2 \mu \mathrm{M}$ was determined using the $\mu$ Quant ${ }^{\mathrm{TM}}$ platewell reader.

A low cost LED-photodiode detector was investigated to compare its performance with that of the PEDD. As shown in Fig. S5 the mean change in absorbance, i.e. peak height was plotted against malachite green-molybdphosphate complex concentration (C) resulting in a dynamic range of $0.9-10 \mu \mathrm{M}$. A linear range of $\left(\mathrm{R}^{2}\right.$ value 0.9816$)$ of $2-$ $10 \mu \mathrm{M}$ was achieved, with an R.S.D. $(n=3)$ of $6.4 \%$. A significantly higher LOD of 2 $\mu \mathrm{M}$ was determined using this comparative LED-photodiode detector. 


\section{Conclusions}

We have demonstrated that the novel, low cost, miniaturized PEDD flow analysis system is highly sensitive for the detection of phosphate. Under optimised conditions the PEDD detector offered a linear range of $0.02-2 \mu \mathrm{M}$ and an LOD of $2 \mathrm{nM}$. For comparative purposes a simple, low cost LED-photodiode detector and a $\mu$ Quant ${ }^{\mathrm{TM}}$ platewell reader were investigated. The LED-photodiode detector achieved an LOD in the micromolar range $(2 \mu \mathrm{M})$. Enhanced performance of the LED-photodiode can be achieved by using more precise components and/or higher resolution ADCs, however, the cost incurred for slight increases in sensitivity would not be justified on a low cost sensor. The PEDD device exhibited sensitivity in the nanomolar concentration range, which was approximately 100 times lower than that of the commercially available bench top platewell reader. The PEDD offers advantages of extremely low power consumption, no requirement for an $\mathrm{A} / \mathrm{D}$ converter or operational amplifier and the sensor can be operated from a $9 \mathrm{~V}$ battery. This low cost detector could therefore be used in an autonomous instrument for remote monitoring of phosphate levels in-situ.

\section{Acknowledgements}

The authors wish to thank Science Foundation Ireland SFI for grant support under the Adaptive Information Cluster Award (SFI 03/IN3/1361), and to acknowledge the assistance of Mitsubishi Electric Research Laboratory, Cambridge, $\mathrm{Ma}$, in the development of the PEDD detector concept. 


\section{References}

[1] J.L. Haberer, J.A. Brandes, Marine Chemistry, 82 (2003) 185-196.

[2] A.G. Vlessidis, M.E. Kotti, N.P. Evmiridis, Journal of Analytical Chemistry, 59 (2004) 77-85.

[3] G. Hanrahan, M. Gledhill, P.J. Fletcher, P.J. Worsfold, Analytica Chimica Acta, 440 (2001) 55-62.

[4] K. Higuchi, H. Tamanouchi, S. Motomizu, Analytical Sciences, 14 (1998) 941946.

[5] J.B. Mullin, J.P. Riley, Analytica Chimica Acta, 12 (1955) 162-176.

[6] P.J. Worsfold, L.J. Gimbert, U. Mankasingh, O.N. Omaka, G. Hanrahan, P.C.F.C. Gardolinski, P.M. Haygarth, B.L. Turner, M.J. Keith-Roach, I.D. McKelvie, Talanta, Analysis of Phosphorus in Environmental and Agricultural Samples, 66 (2005) 273-293.

[7] B. Paull, L. Barron, P. Nesterenko, in Chromatographic Analysis of the Environment: Third Ed, Revised and Expanded, CRC Press/Taylor \& Francis Group: Boca Raton, FL., 2005, p. 263-286.

[8] M. Sequeira, M. Bowden, E. Minogue, D. Diamond, Talanta, 56 (2002) 355-363.

[9] M. Bowden, D. Diamond, Sensors and Actuators B: Chemical, 90 (2003) 170174.

[10] D. Peachey, J.L. Roberts, J. Scot-Baker, Journal of Geochemical Exploration, 2 (1973) 115-120.

[11] E. Bakker, D. Diamond, A. Lewenstam, E. Pretsch, Analytica Chimica Acta, 393 (1999) 11-18. 
[12] K.L. Linge, C.E. Oldham, Analytica Chimica Acta, 450 (2001) 247-252.

[13] H.H. Hess, J.E. Derr, Analytical Biochemistry, 63 (1975) 607-613.

[14] P.P. Van Veldhoven, G.P. Mannaerts, Analytical Biochemistry, 161 (1987) 45-48.

[15] P. Ekman, O. Jager, Analytical Biochemistry, 214 (1993) 138-141.

[16] S. Motomizu, T. Wakimoto, K. Tôei, The Analyst, 108 (1983) 361-367.

[17] J.P. Susanto, M. Oshima, S. Motomizu, H. Mikasa, Y. Hori, The Analyst, 120 (1995) 187-191.

[18] I.D. McKelvie, D.M.W. Peat, P.J. Worsfold, Analytical Proceedings, 32 (1995) 437-445.

[19] E. D'Angelo, J. Crutchfield, M. Vandiviere, J Environ Qual, 30 (2001) 22062209.

[20] S. Motomizu, Z.-H. Li, Talanta, Analysis of Phosphorus in Environmental and Agricultural Samples, 66 (2005) 332-340.

[21] P.K. Dasgupta, I.-Y. Eom, K.J. Morris, J. Li, Analytica Chimica Acta, 500 (2003) 337-364.

[22] A. Zukauskas, M.S. Shur, R. Gaska, Introduction to Solid-State Lighting, John Wiley \& Sons, Inc., 2002.

[23] Y. Taniyasu, M. Kasu, T. Makimoto, Nature, 441 (2006) 325-328.

[24] O. Geschke, H. Klank, P. Tellemann, Microsystem Engineering of Lab-on-a-chip Devices, WILEY-VCH, 2004.

[25] M. O' Toole, K.T. Lau, D. Diamond, Talanta, 66 (2005) 1340-1344.

[26] M. O’ Toole, K.-T. Lau, B. Shazmann, R. Shepherd, P.N. Nesterenko, B. Paull, D. Diamond, The Analyst, 131 (2006) 938 - 943. 
[27] L. Barron, P.N. Nesterenko, D. Diamond, M. O’Toole, K.T. Lau, B. Paull, Analytica Chimica Acta, 577 (2006) 32-37.

[28] D. Diamond, Analytical Chemistry, 76 (2004) 279A-286A.

[29] K.-T. Lau, S. Baldwin, M. O'Toole, R. Shepherd, W.J. Yerazunis, S. Izuo, S. Ueyama, D. Diamond, Analytica Chimica Acta, 557 (2006) 111-116.

[30] T. Attin, K. Becker, C. Hannig, W. Buchalla, A. Wiegand, Clinical Oral Investigations, 9 (2005) 203-207. 


\section{List of Figures}

Figure 1 A schematic of the integrated PEDD flow analysis device used for colorimetric detection.

Figure 2 Emission spectrum $\left(\lambda_{\max } 636 \mathrm{~nm}\right)$ of the emitter LED (dashed line) used in the integrated PEDD flow analysis device, the absorption spectrum $\left(\lambda_{\max } 640 \mathrm{~nm}\right)$ of $0.9 \mu \mathrm{M} \mathrm{PO}_{4}$ and MG reagent (solid line) and $\left(\lambda_{\max } 450 \mathrm{~nm}\right) \mathrm{MG}$ reagent (bold dashed line).

Figure 3 Kinetic study of the colour formation between $0.5 \mu \mathrm{M} \mathrm{PO}_{4}$ and $\mathrm{MG}$ reagent $(\mathbf{n}=3)$.

Figure 4 Log of the discharge times (t) obtained using a PEDD versus malachite green-molybdphosphate complex concentration. The error bars represent the standard deviations for $n=3$. The inset shows the dynamic range of responses obtained from the calibration.

Figure 5 Determination of the LOD of the malachite green-molybdphosphate complex concentration $(2 \mathrm{nM})$.

Figure S1 Electronic circuitry of the LED-photodiode detector. Capacitor $(\mathrm{C1})=10$ pF, LED $(D 1)=$ Red LED, Photodiode $(D 2)=\operatorname{IPL10020BW}$, Resistor $($ R1 $)=10 \mathrm{~K} \Omega$, R2 = $40 \mathrm{M} \Omega$ and Operational Amplifier (IC1) = CA3140.

Figure S2 Determination of optimum reagent to sample $\left(2 \mu \mathrm{M} \mathrm{PO}_{4}\right)$ ratio. The error bars represent the standard deviations for $n=3$.

Figure S3 Plot obtained for $0.5 \mu \mathrm{M} \mathrm{PO}_{4} \mathrm{MG}$ reagent sample using $0.004 \mathrm{k} \Omega$ (black line), $1.58 \mathrm{k} \Omega$ (bold line) and $6.02 \mathrm{k} \Omega$ (bold dashed line). 
Figure S4 Calibration plot of absorbance at $\lambda_{\max }$ obtained using a $\mu$ Quant ${ }^{\mathrm{TM}}$ platewell reader versus malachite green-molybdphosphate complex concentration. The error bars represent the standard deviations for $n=3$.

Figure S5 Calibration plot of absorbance obtained using an LED-photodiode detector versus malachite green-molybdphosphate complex concentration. The error bars represent the standard deviations for $n=3$. 
Figure 1

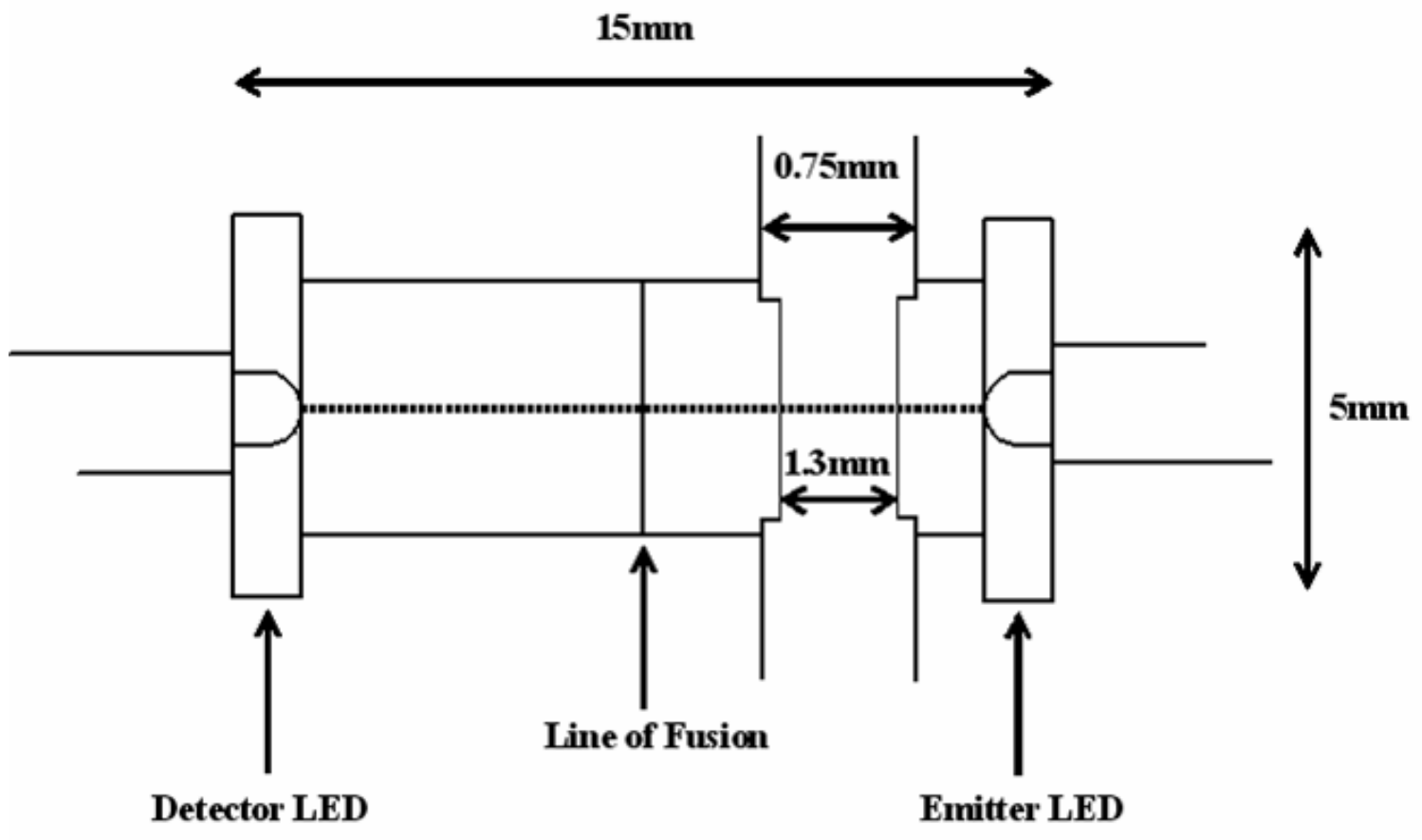


Figure 2

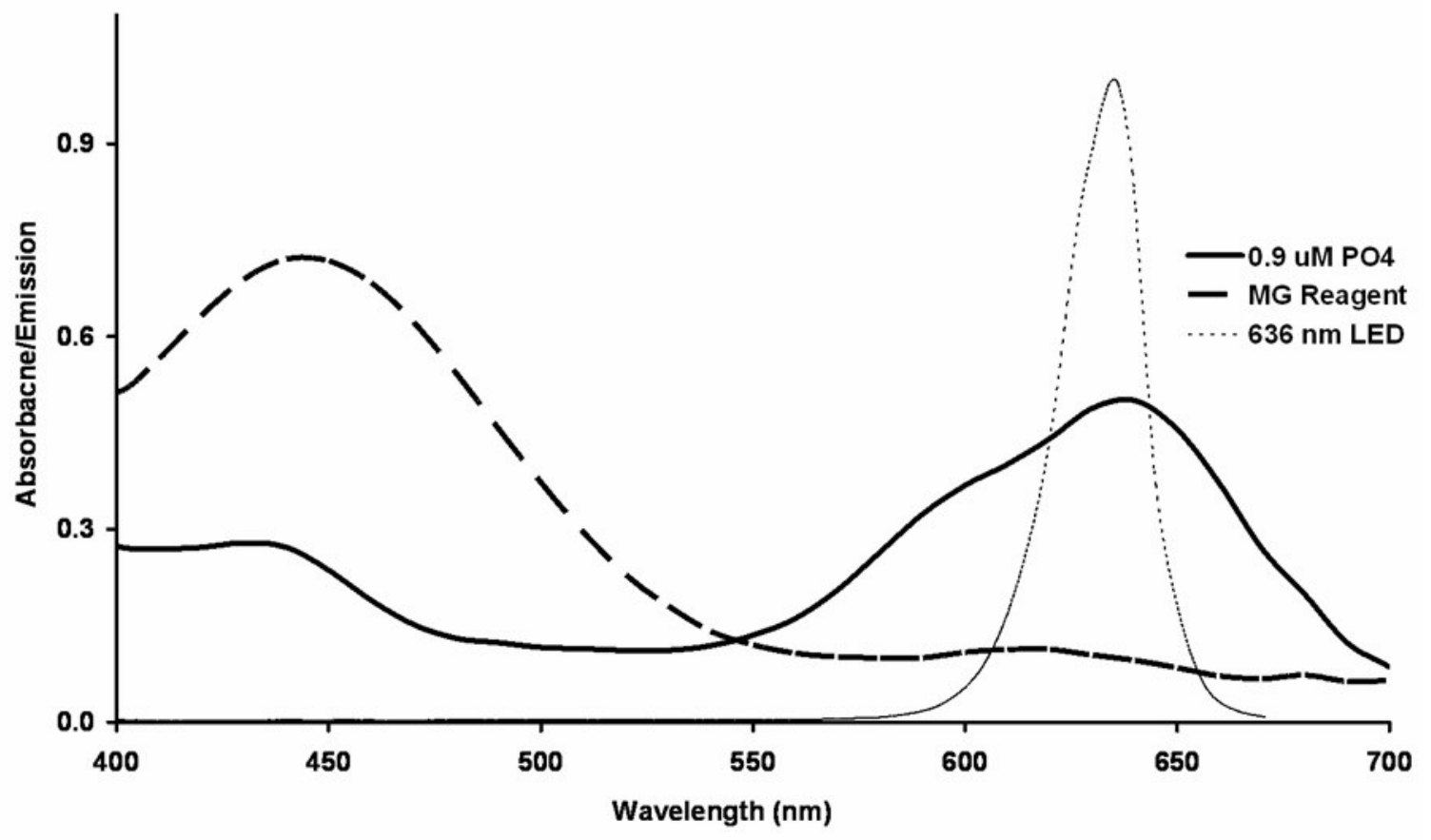


Figure 3

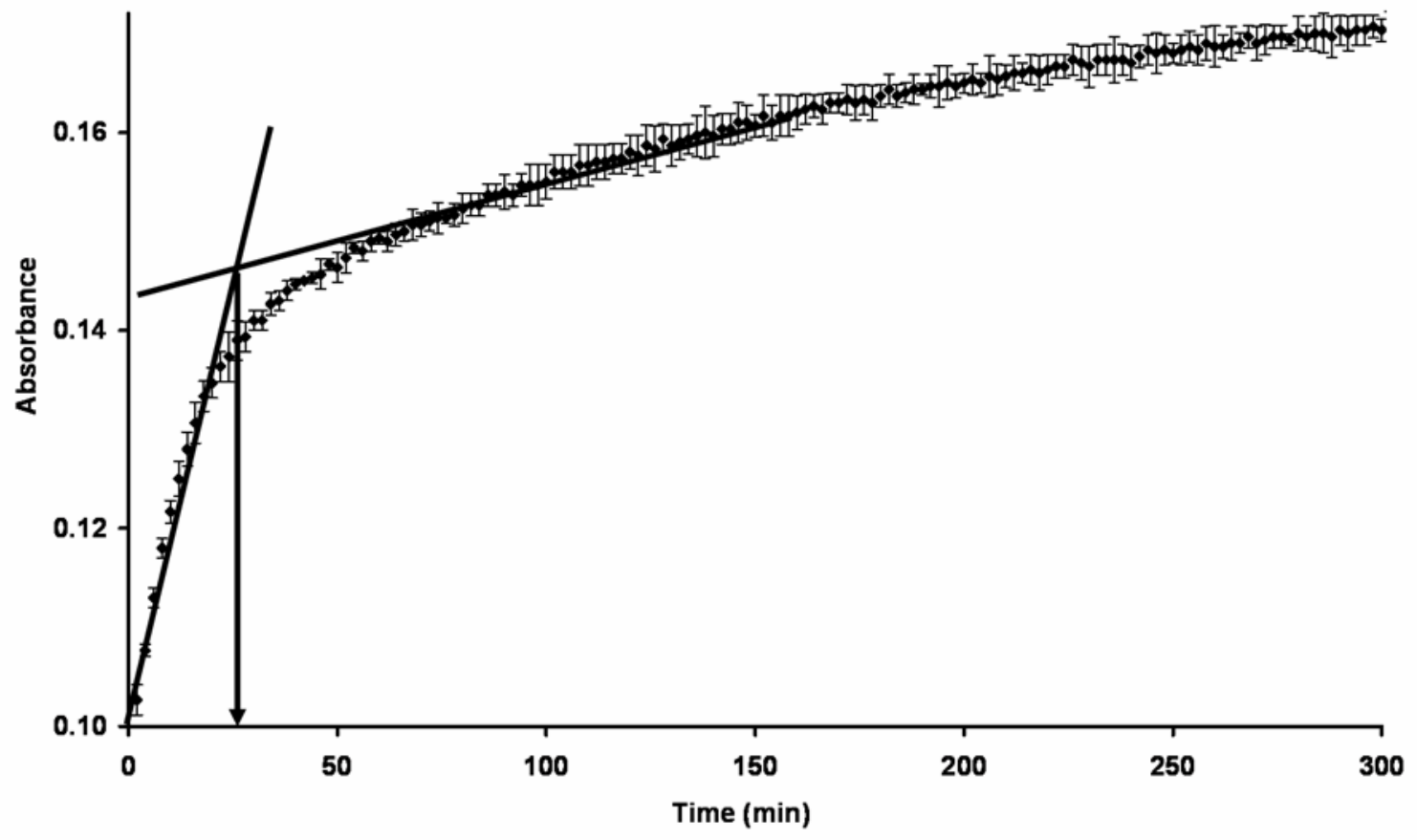


Figure 4

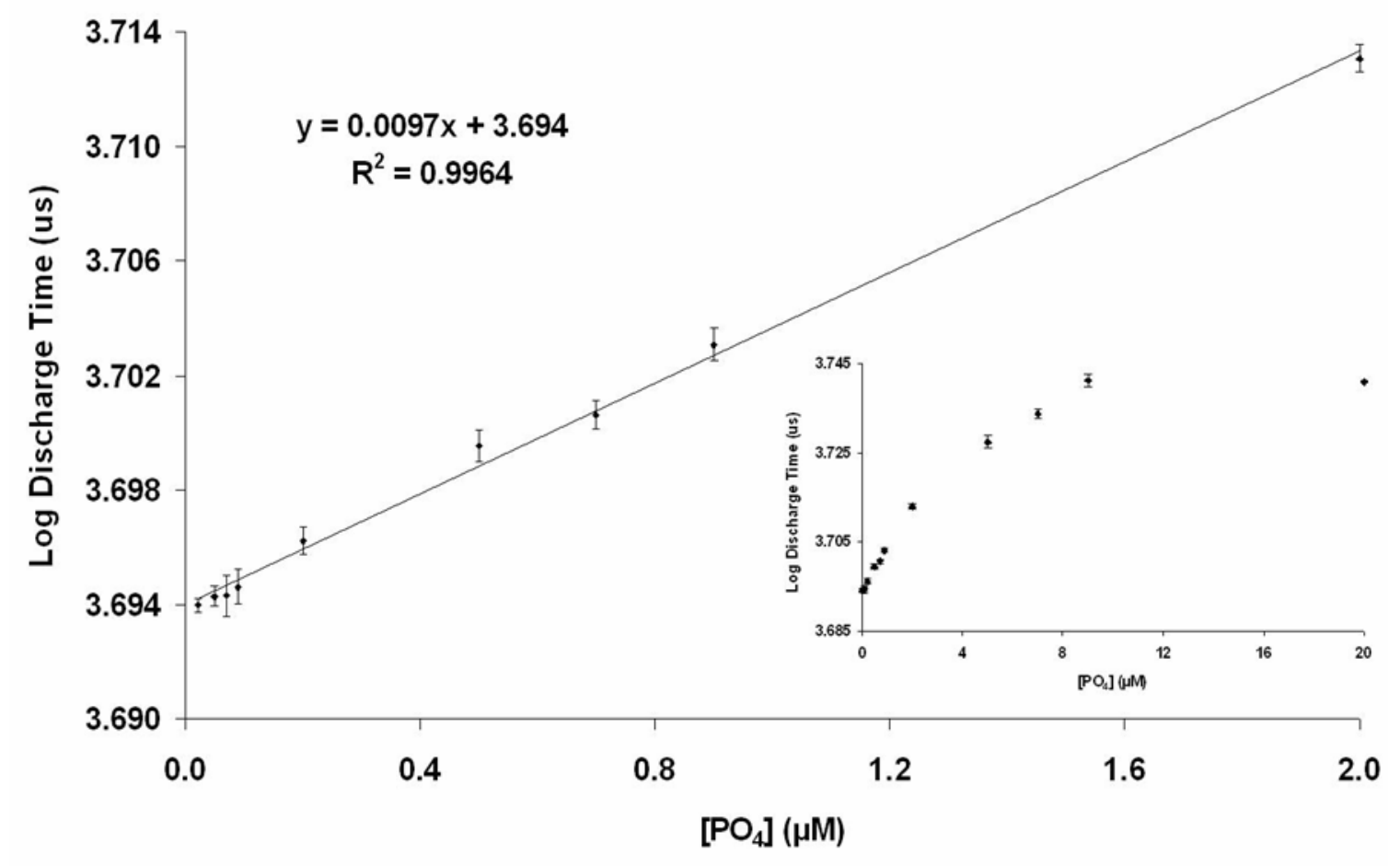


Figure 5

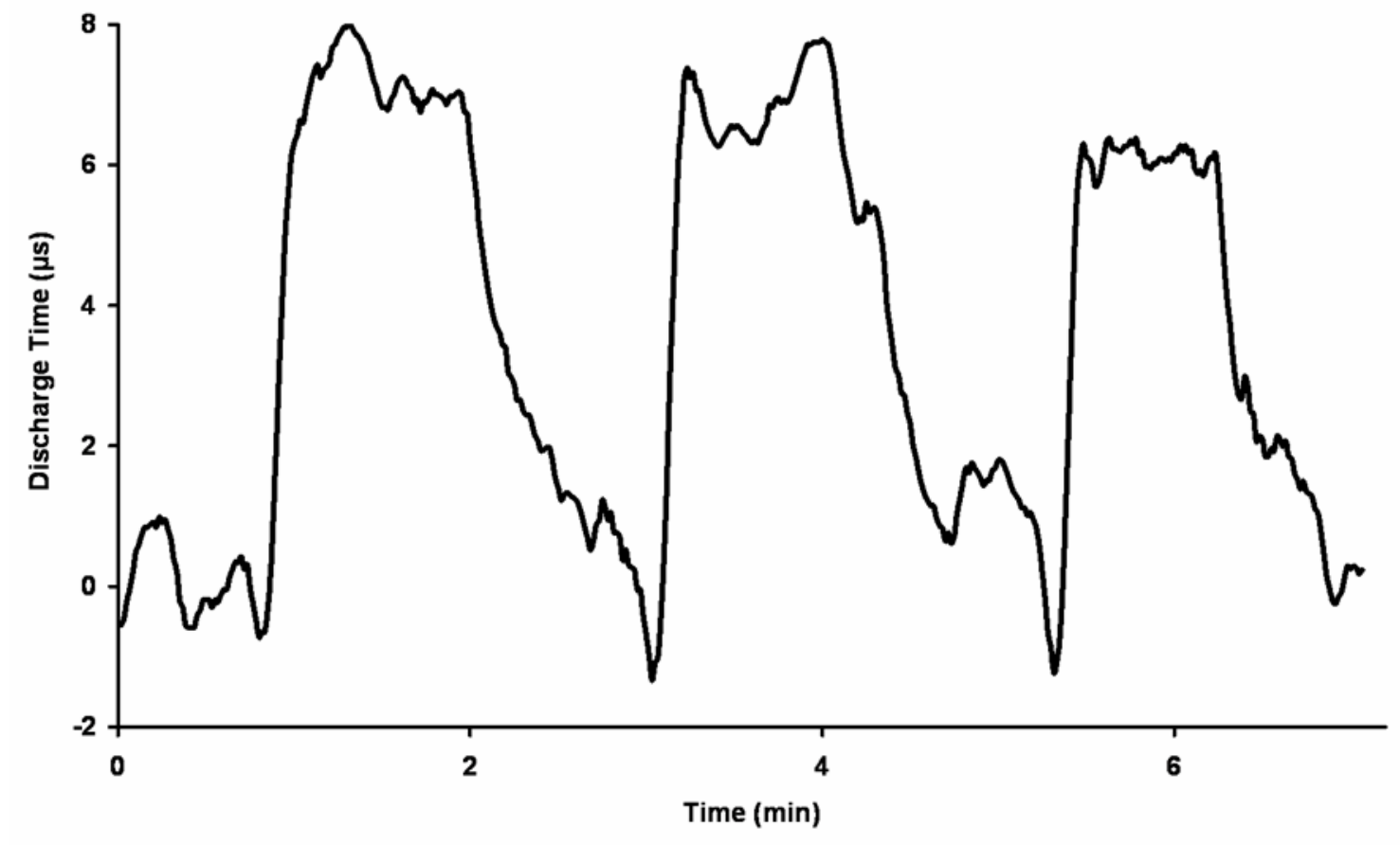


Figure S1

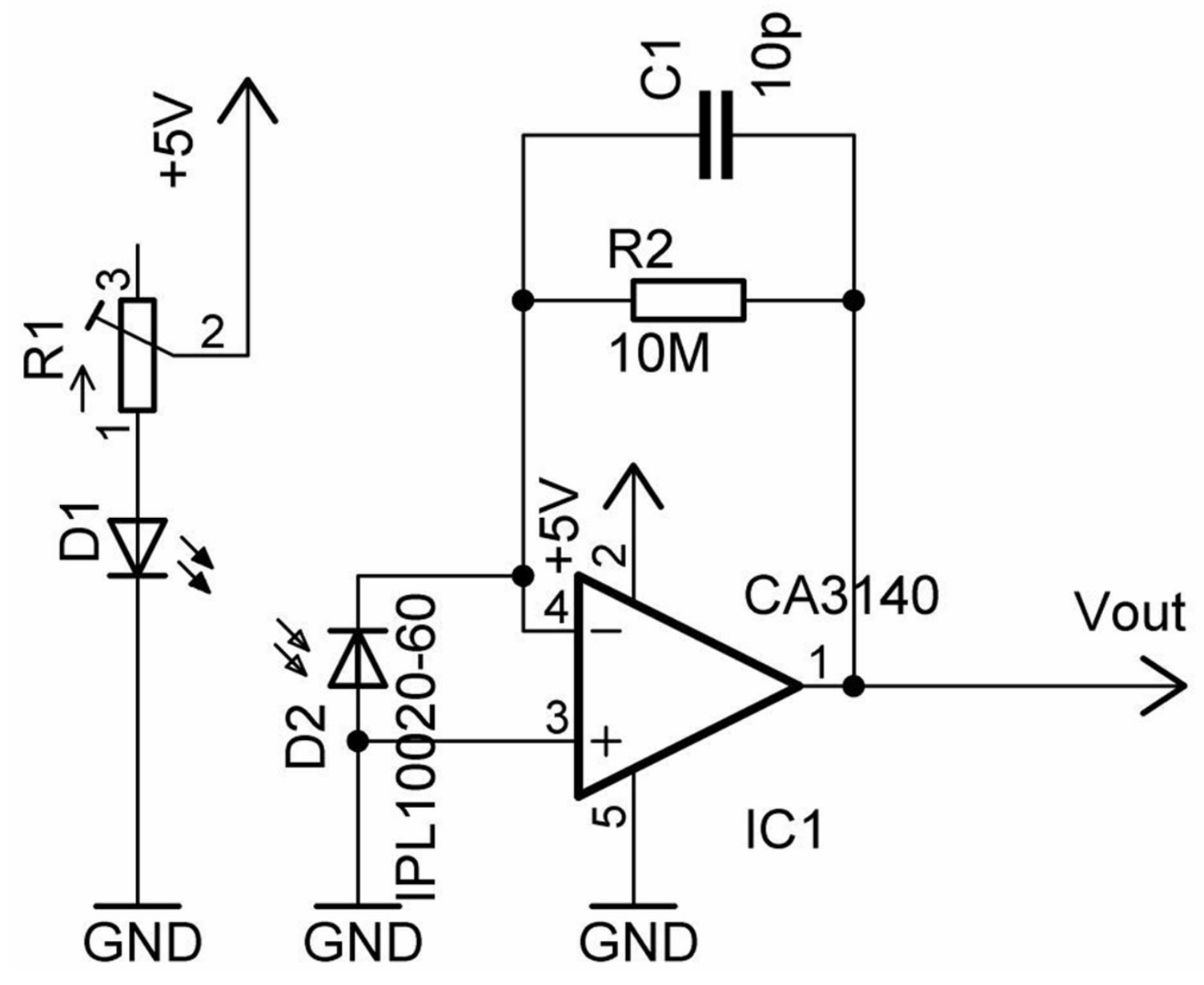


Figure S2

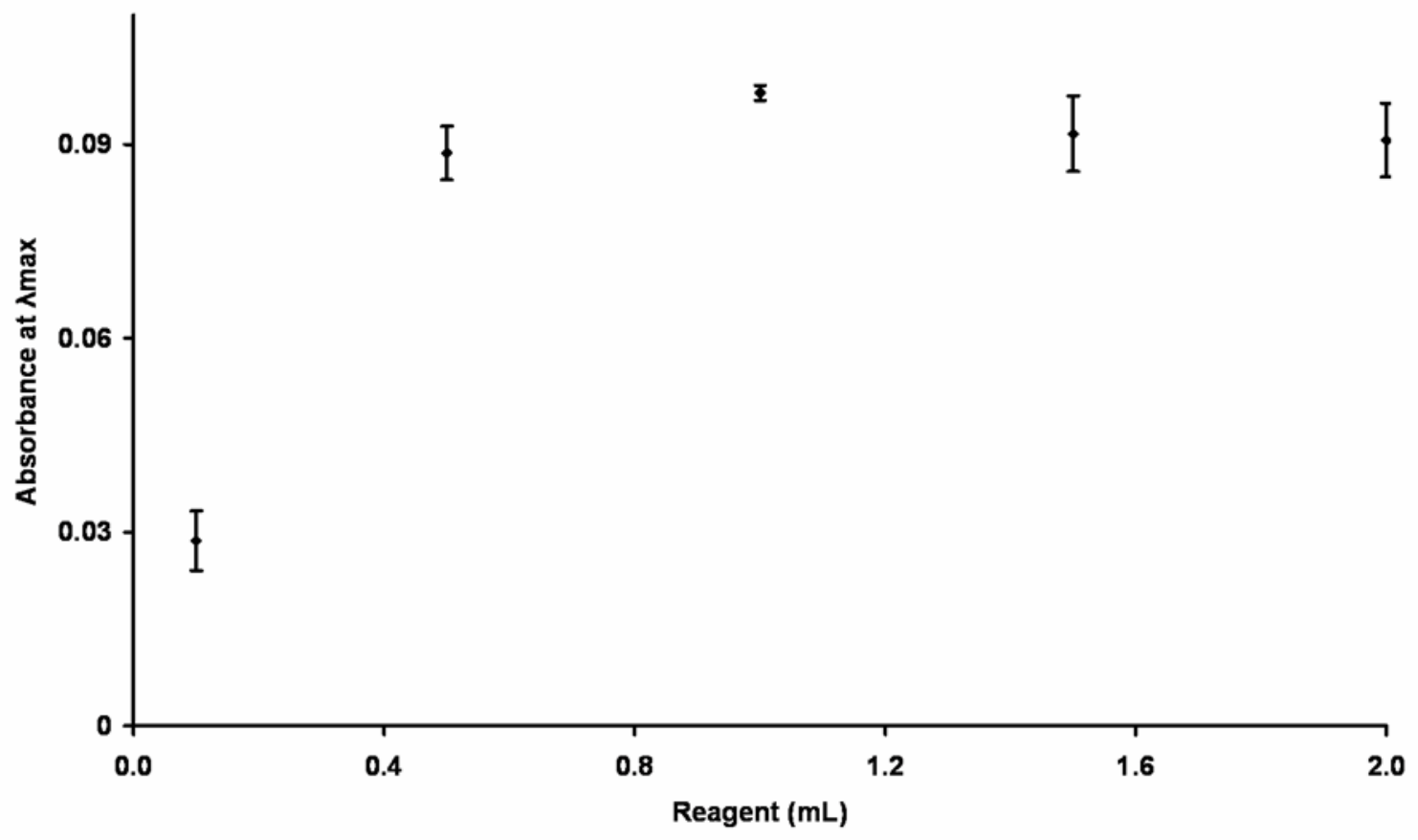


Figure S3

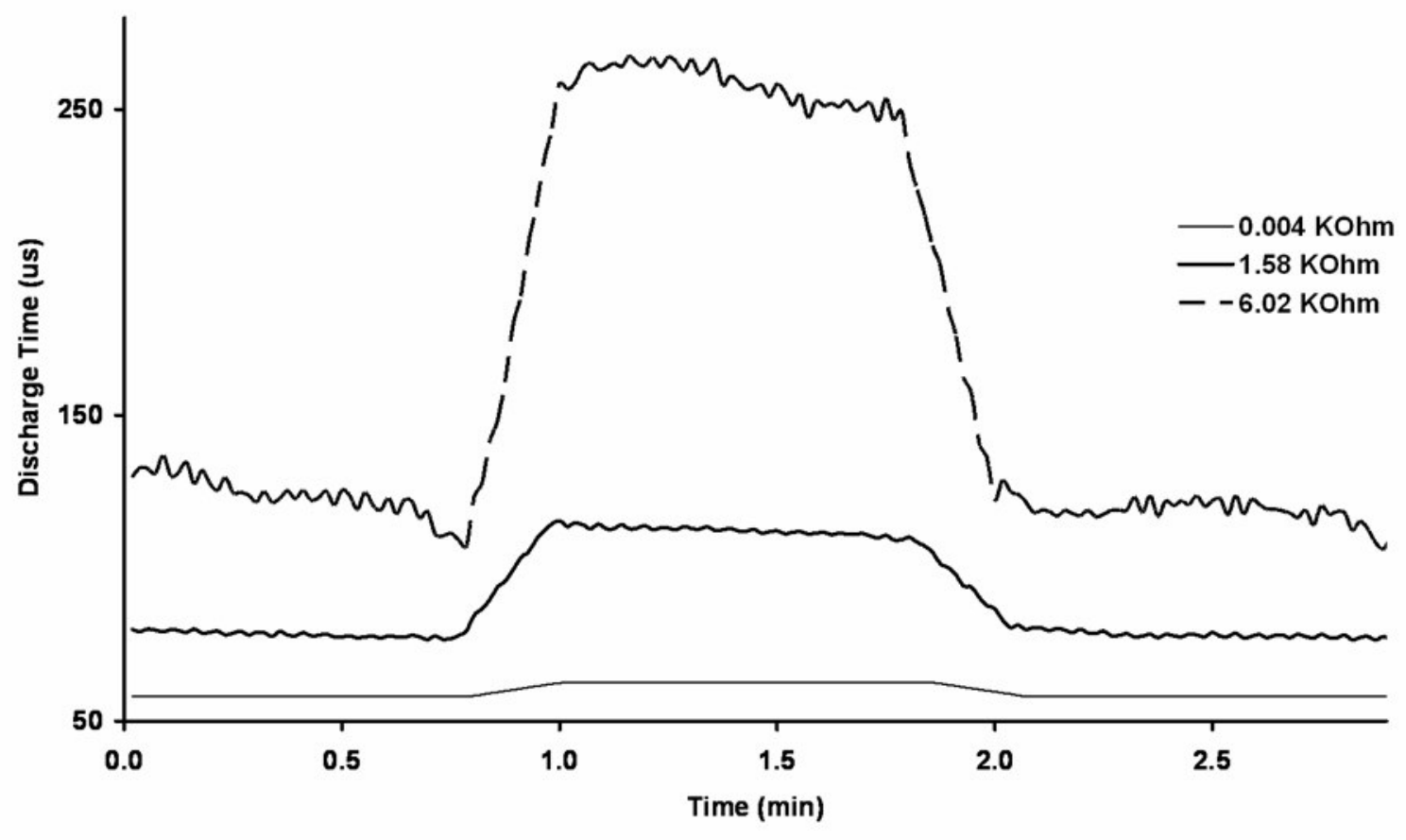




\section{Figure S4}

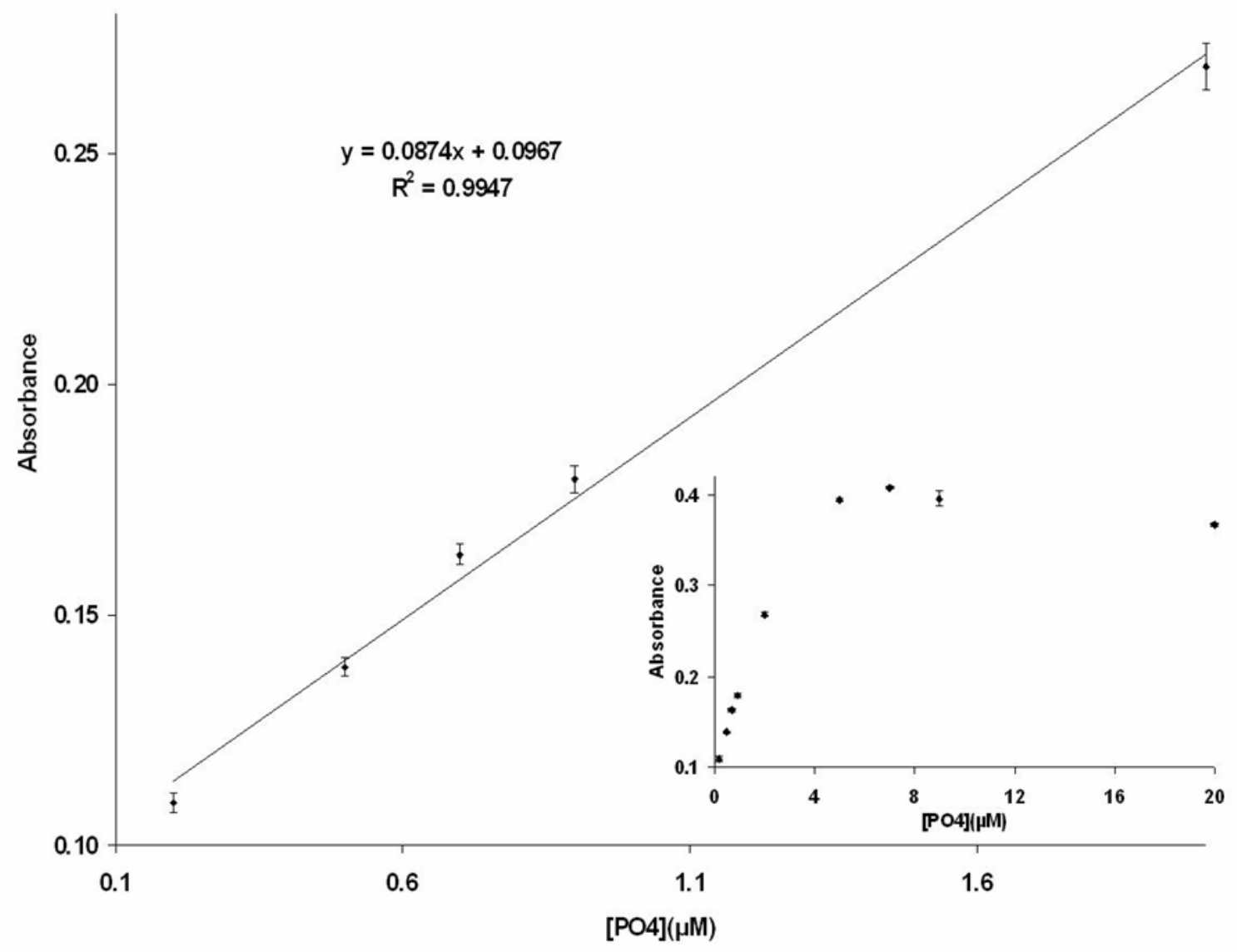




\section{Figure S5}

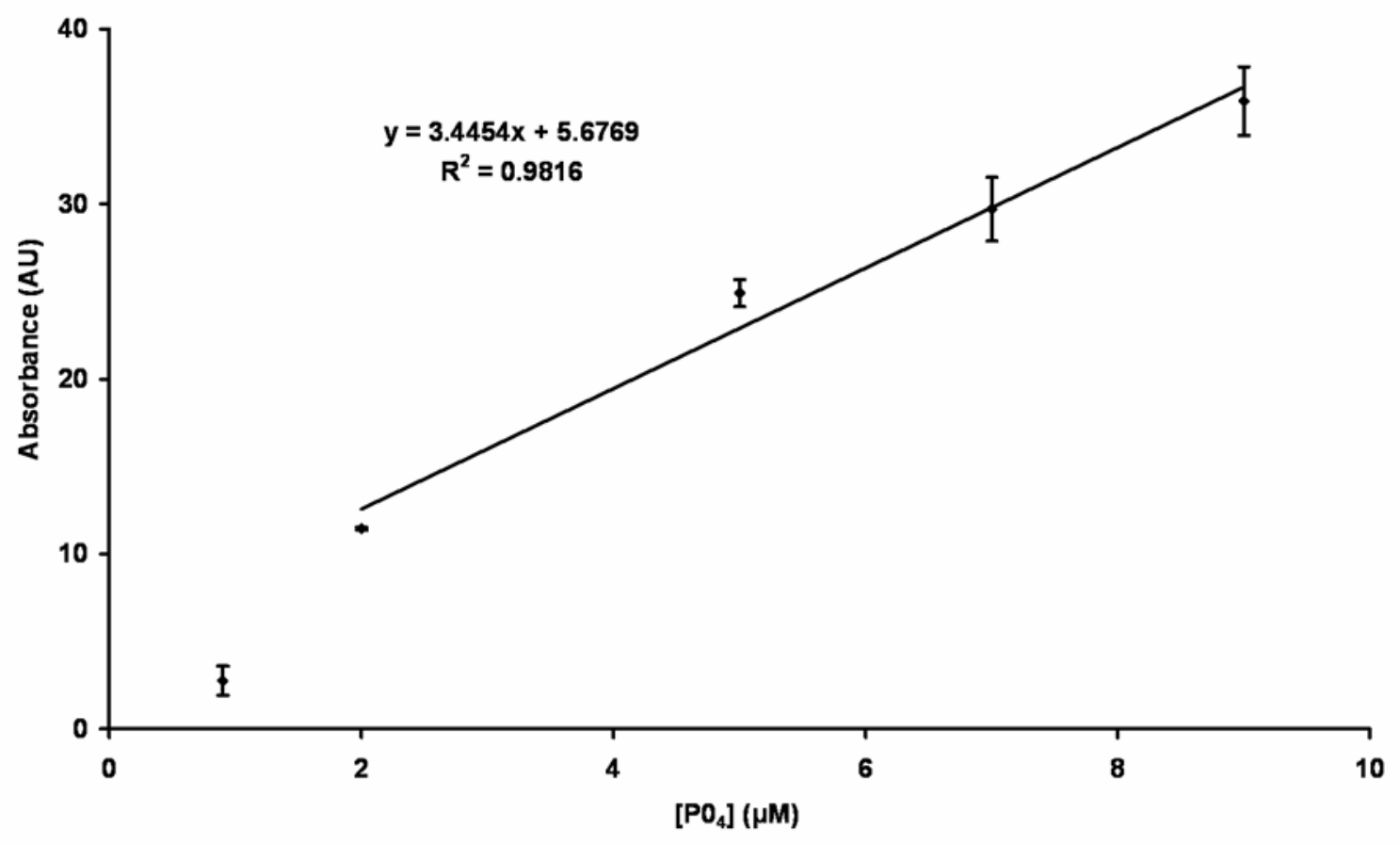

